\title{
473653 - OXIDATIVE STRESS MARKERS AND POSTOPERATIVE HYPERTENSION IN PATIENTS UNDERGOING NEUROSURGERY - A CORRELATIVE STUDY
}

Sanjib Adhikary, M.D. ${ }^{1}$, Parthiban Velayutham, Msc. ${ }^{2}$, Anup Ramchandran, Phd ${ }^{3}$, Rajsekhar Vedantum, Mch ( Neurosurgery) ${ }^{2}$, Srinivas Babu, Phd ${ }^{2}$

1. Anaesthesia, Christian Medical College, Vellore, India

2. Neurological Sciences, Christian Medical College, Vellore, India

3. Wellcome Trust Research Lab, Christian Medical College, Vellore, India

Introduction: Postoperative hypertension after craniotomy is a common problem even when compared to other surgeries like cardiac surgeries (1). Correlation of vasoactive modulators in relation to increased stress response in Craniotomy has already been studied. (1) The role of oxidative stress in cellular damage in animal models of surgically induced brain injury has been investigated (2), but information from humans, is sparse. In this exploratory observational study, we examine the temporal profile of oxidative stress markers in patients undergoing supratentorial craniotomy procedures and correlate this to postoperative hypertension.

Methods: After IRB approval, written consent was obtained from 16 patients. 3ml of blood was collected from all the patients for the measurement of oxidative stress markers \{malondialdehyde (MDA), protein carbonyl, and nitrate at different stated intervals. A standard protocol for anesthesia was used. Hypertension was defined as mean blood pressure more than $20 \%$ of the preoperative value.The arterial blood pressure was recorded in a computerized monitor at 5-minute intervals through out the study period. The data was then compared statistically using Student's independent sample t-test and Pearson's correlation co-efficient wherever necessary.

Results: On the basis of mean difference of blood pressure between pre operative and at extubation period 11 patients had hypertension and 5 patients were normotensive. The mean \pm SD of oxidative stress markers and mean arterial pressure (MAP) in hypertensive and Normotensive patients were shown in the table

Discussion: This study demonstrates that oxidative stress markers are elevated both during surgery and in the immediate postoperative period in a number of patients undergoing supratentorial craniotomies. This increase in markers correlates with their MAP. Further studies are in progress to determine if whether the increase in oxidative stress markers is a cause of effect of increased sympathetic activity causing postoperative hypertension.

References: 1.Olsen KS, Pedersen CB, Madsen JB, Ravn LI, Schifter S. Vasoactive modulators during and after craniotomy: relation to postoperative hypertension. Journal of neurosurgical anesthesiology 2002;14(3):171-9. 2. Cirak B, Inci S, Palaoglu S, Bertan V. Lipid peroxidation in cerebral tumors. Clinica chimica acta; international journal of clinical chemistry 2003;327(1-2):103-7. 


\begin{tabular}{|c|c|c|c|c|c|c|}
\hline 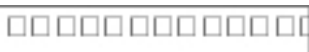 & 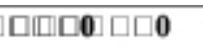 & $\square \square \square \mathbf{0} \square$ & $\mathbf{0} \square \square \square \square \mathbf{0} \square \square \square \mathbf{0}$ & 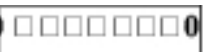 & \multirow[b]{3}{*}{ (ㅁ) } & \multirow[b]{3}{*}{ प्मा } \\
\hline 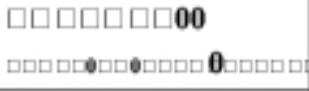 & $\square$ & $\square \square \square \mathbf{D \square} \square$ & 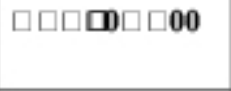 & $\square \square \square \mathbf{D \square} \square \mathbf{0}$ & & \\
\hline \multirow{2}{*}{ 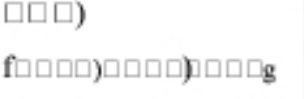 } & 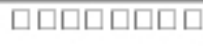 & 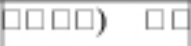 & 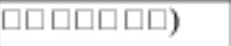 & 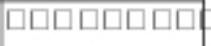 & & \\
\hline & 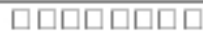 & 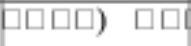 & 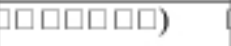 & 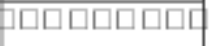 & ) & पᄆ口ロI \\
\hline \multirow{2}{*}{ 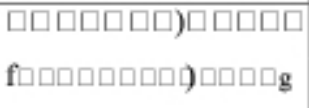 } & 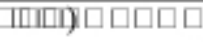 & 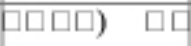 & 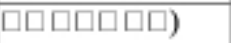 & 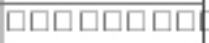 & (口) & प्म[ \\
\hline & 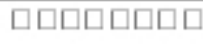 & 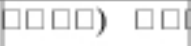 & पिएवप्वप) & 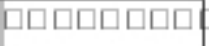 & 7) & प्म० \\
\hline \multirow{2}{*}{ 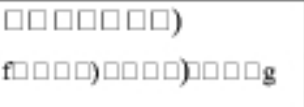 } & 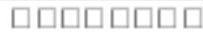 & ロロロロ) & 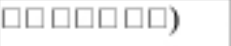 & 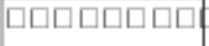 & \multirow[t]{2}{*}{ () } & प्मट \\
\hline & 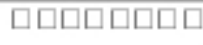 & 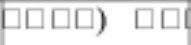 & 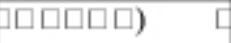 & 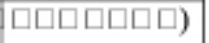 & & ロロロロロ \\
\hline \multirow{3}{*}{ 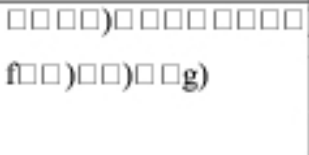 } & 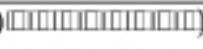 & 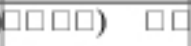 & प्म) & (प口) & \multirow{3}{*}{\multicolumn{2}{|c|}{ 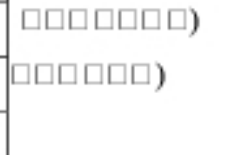 }} \\
\hline & 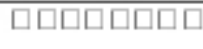 & 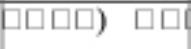 & प्प & () & & \\
\hline & \multicolumn{4}{|c|}{ 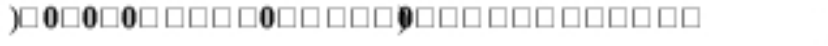 } & & \\
\hline
\end{tabular}

